\title{
Article
}

\section{Pilot Study Comparing Closed Versus Open Tracheal Suctioning in Postoperative Neonates and Infants With Complex Congenital Heart Disease}

\author{
Tume, Lyvonne Nicole, Baines, Paul B., Guerrero, Rafael, Hurley, \\ Margaret Anne, Johnson, Robert, Kalantre, Atul, Ramaraj, Ram, \\ Ritson, Paul C., Walsh, Laura and Arnold, Philip D. \\ Available at http://clok.uclan.ac.uk/18301/ \\ Tume, Lyvonne Nicole ORCID: 0000-0002-2547-8209, Baines, Paul B., \\ Guerrero, Rafael, Hurley, Margaret Anne ORCID: 0000-0002-2502-432X, \\ Johnson, Robert, Kalantre, Atul, Ramaraj, Ram, Ritson, Paul C., Walsh, Laura et \\ al (2017) Pilot Study Comparing Closed Versus Open Tracheal Suctioning in \\ Postoperative Neonates and Infants With Complex Congenital Heart Disease. \\ Pediatric Critical Care Medicine, 18 (7). pp. 647-654. ISSN 1529-7535
}

It is advisable to refer to the publisher's version if you intend to cite from the work. http://dx.doi.org/10.1097/PCC.0000000000001192

For more information about UCLan's research in this area go to http://www.uclan.ac.uk/researchgroups/ and search for <name of research Group>.

For information about Research generally at UCLan please go to http://www.uclan.ac.uk/research/

All outputs in CLoK are protected by Intellectual Property Rights law, including Copyright law. Copyright, IPR and Moral Rights for the works on this site are retained by the individual authors and/or other copyright owners. Terms and conditions for use of this material are defined in the policies page. 


\title{
Pilot Study Comparing Closed Versus Open Tracheal Suctioning in Postoperative Neonates and Infants With Complex Congenital Heart Disease
}

\author{
Lyvonne N. Tume, RN, PhD ${ }^{1,2}$; Paul B. Baines, MD, PhD, MRCP, FRCA, FFICM ${ }^{1}$; \\ Rafael Guerrero, FRCSI, FRCS (CTh), FETCS, $\mathrm{MD}^{3}$; Margaret A. Hurley, $\mathrm{PhD}^{2}$; \\ Robert Johnson, BM, $\mathrm{MRCP}^{3}$; Atul Kalantre, $\mathrm{MBBS} \mathrm{MRCPCH}^{3}$; \\ Ram Ramaraj, MBBS, DCH, MRCPCH ${ }^{3}$; Paul C. Ritson, MCSP' \\ Laura Walsh, RNC, BSc (Hons) ${ }^{1}$; Philip D. Arnold, BM, FRCA ${ }^{3}$
}

\begin{abstract}
Objectives: To determine the hemodynamic effect of tracheal suction method in the first 36 hours after high-risk infant heart surgery on the PICU and to compare open and closed suctioning techniques.

Design: Pilot randomized crossover study.

Setting: Single PICU in United Kingdom.

Participants: Infants undergoing surgical palliation with Norwood Sano, modified Blalock-Taussig shunt, or pulmonary artery banding in the first 36 hours postoperatively.
\end{abstract}

Interventions: Infants were randomized to receive open or closed (in-line) tracheal suctioning either for their first or second study tracheal suction in the first 36 hours postoperatively.

Measurements and Main Results: Twenty-four infants were enrolled over 18 months, 11 after modified Blalock-Taussig shunt, seven after Norwood Sano, and six after pulmonary artery banding. Thirteen patients received the open suction method first

'PICU, Alder Hey Children's NHS Foundation Trust, Liverpool, United Kingdom.

${ }^{2}$ University of Central Lancashire, College of Health \& Wellbeing, Preston, United Kingdom.

${ }^{3}$ Alder Hey Children's NHS Foundation Trust, Liverpool, United Kingdom. This study was undertaken at PICU Alder Hey Children's NHS Foundation Trust.

Supported, in part, by Alder Hey Children's Hospital Charitable Funds (PICU donated) and the Hemby Trust to the value of $£ 70,000$.

Dr. Tume disclosed that the Alder Hey Children's Hospital Charity and the Hemby Trust funded this research to the value of $£ 70,000$. Dr. Hurley's institution received funding $(£ 2,500)$ from Alder Hey Hospital Charity for Dr. Hurley to complete the statistical analysis of the data and to prepare tables and figures for the article. Mr. Johnson received support for article research from the Alder Hey Charity, and his institution received funding from the Alder Hey Charity. Mr. Kalantre received support for article research from the Alder Hey Charity, and his institution received funding from the Alder Hey Charity. The remaining authors have disclosed that they do not have any potential conflicts of interest.

For information regarding this article, E-mail: Lyvonne.tume@alderhey.nhs.uk Copyright (C) 2017 by the Society of Critical Care Medicine and the World Federation of Pediatric Intensive and Critical Care Societies

DOI: $10.1097 /$ PCC.0000000000001192 followed by the closed suction method second, and 11 patients received the closed suction method first followed by the open suction method second in the first 36 hours after their surgery. There were statistically significant larger changes in heart rate $(p=0.002)$, systolic blood pressure $(p=0.022)$, diastolic blood pressure $(p=0.009)$, mean blood pressure $(p=0.007)$, and arterial saturation $(p=0.040)$ using the open suction method, compared with closed suctioning, although none were clinically significant (defined as requiring any intervention).

Conclusions: There were no clinically significant differences between closed and open tracheal suction methods; however, there were statistically significant greater changes in some hemodynamic variables with open tracheal suctioning, suggesting that closed technique may be safer in children with more precarious physiology. (Pediatr Crit Care Med 2017; XX:00-00)

Key Words: cardiac intensive care; child; pediatric critical care; single ventricle physiology; suction

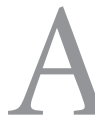

lthough the survival rates have improved for infant congenital cardiac surgery, three operations continue to be associated with high early postoperative mortality: Norwood Sano (NS) procedure, systemic to pulmonary artery shunt, and pulmonary artery bands (PABs) (1). The first 48 hours after surgery is a high-risk period. A major reason for such vulnerability may be the unstable relationship created between pulmonary and systemic blood flow, along with postoperative myocardial dysfunction and low cardiac output.

Tracheal suctioning (ETS) is an essential procedure for any intubated child to remove secretions and prevent tube occlusion (2-4). ETS is known to potentially provoke hemodynamic instability in these infants and therefore is considered a high-risk procedure (5). To date, there is no published work on the hemodynamic impact of ETS in this specific group of high-risk infants, and neither open nor closed (in-line) suctioning is known to be 
superior. Evidence in preterm and term ventilated infants with respiratory disease shows that closed suction may promote more rapid recovery, less hypoxia, less bradycardia, fewer changes in systolic blood pressure (SBP), and less lung volume loss $(3,6-8)$. However, others have shown that closed circuit was less effective at removing secretions (9) and that it impaired ventilator function when a volume-targeted mode was used (10). We aimed to determine the effect of tracheal suction method in the first 36 hours after high-risk infant heart surgery on the PICU and to compare open and closed suctioning techniques in these infants.

\section{MATERIALS AND METHODS}

A randomized crossover pilot study was undertaken in a single PICU in the United Kingdom. Infants were randomized to receive either open or closed tracheal suction either first or second in the first 36 -hour period postoperatively. The specific study objectives were as follows:

1) To describe the changes that occur in hemodynamic and respiratory variables and cerebral near infrared spectroscopy (NIRS) during ETS in the first 36 hours after surgery.

2) To evaluate shunt and pulmonary artery blood flow velocity using echocardiography before, during, and after tracheal suction.

3) To determine the infants recovery time (to baseline values) after ETS.

4) To compare the impact of closed versus open tracheal suction procedure in terms of hemodynamic and respiratory variables and cerebral NIRS impact.

\section{Participants}

Between September 2014 and January 2016, all infants undergoing high-risk cardiac surgery as defined below and whose parents consented for the study were included. Infants requiring surgery for complex congenital heart disease undergoing three specific surgical procedures: Norwood or NS operation, a $\mathrm{PAB}$, or a modified Blalock-Taussig shunts (MBTSs) who were sedated and muscle-relaxed in the first 36 hours after surgery were included. Patients were excluded if the parents refused consent, the child required extracorporeal membrane oxygenation (ECMO), or the child was not sedated and muscle relaxed.

The randomization sequence was generated using sealedenvelope.com (11) and was converted into sealed envelopes by the chief investigator (L.N.T.).

\section{Data Collection}

Two nonurgent ETS episodes were undertaken, and detailed observations were made by an independent observer (research nurse) for the first 36 hours after surgery. Data from the Phillips Intellivue monitor (Phillips, Surrey, United Kingdom) and Foresight Casmed NIRS device (CAS Medical Systems, Branford, CT) were downloaded onto a research laptop and annonymized for analysis. Heart rate, blood pressure (diastolic, systolic, and mean), cerebral NIRS, arterial oxygen saturation, and end-tidal $\mathrm{CO}_{2}$ were recorded at baseline, continuously during the intervention and until the recovery of baseline values.
Echocardiographic measurements of shunt and PAB velocity flow were undertaken 1 minute pre, during, and 1 minute postsuction by three experienced cardiologists using an agreed measurement protocol. Ventilatory variables and arterial blood gases were recorded before and after the procedure.

The defined time points for analysis were as follows: 1) baseline, defined as 1 minute prior to starting suction; 2) peak (averaged) change in variables during the procedure; and 3) postprocedure time (defined as $1 \mathrm{~min}$ after completing suctioning). Recovery time was defined as the time taken for a variable returning to its baseline value after ETS. The study ETS procedure followed reflected our current PICU guidelines for ETS of these infants (standard practice) (Fig. 1). Three senior respiratory physiotherapists carried out all suctioning, with three experienced cardiologists undertaking echocardiograms and experienced cardiac PICU nurses following a detailed protocol (Table 1).

The outcome measures for this study were as follows:

1) The mean change from baseline to maximum in all variables during and post-ETS.

2) The change in echocardiographic variables (shunt velocity, $\mathrm{PAB}$ velocity in $\mathrm{m} / \mathrm{s}$ ) during the procedure.

3) Time to recovery to baseline values (in min) of all variables.

4) The difference in variable change between open and closed ETS.

Infants were all mechanically ventilated with pressure-cycled ventilation (biphasic positive airway pressure mode) using the Drager Evita XL ventilator (Drager, Lubeck, Germany), and pre- and postventilation variables were recorded and organ dysfunction (Pediatric Logistic Organ Dysfunction Score) and Vasoactive Inotrope Scores were recorded in the first 48 hours. Infants were followed up for 30 days or until hospital discharge. The occurence of any serious adverse events (SAEs) such as cardiopulmonary arrest (CPA) defined as the need for active chest compressions, need for emergency ECMO, emergency chest re-exploration, or acute kidney injury requiring peritoneal dialysis were recorded.

Ethical approval was sought and granted by Liverpool East NHS Ethics committee September 2014 (Reference 14/ NW/1071), and written informed consent was taken from all parents prior to patient enrollment.

\section{Analysis}

Data were first analyzed descriptively using standard summary statistics. As we were primarily interested in change over time, change between baseline and peak and change between baseline and postprocedure were defined as the outcomes of interest and were analyzed using a two-strata analysis of variance (ANOVA). The primary objective of the ANOVA was to test whether there were real differences between the open and closed suction procedures in terms of a difference in mean change from baseline to peak and baseline to postprocedure. The interaction between suction method and order of randomization was first tested at the between patient stratum, and subsequently, the main effects of order of randomization and suction method were tested at 


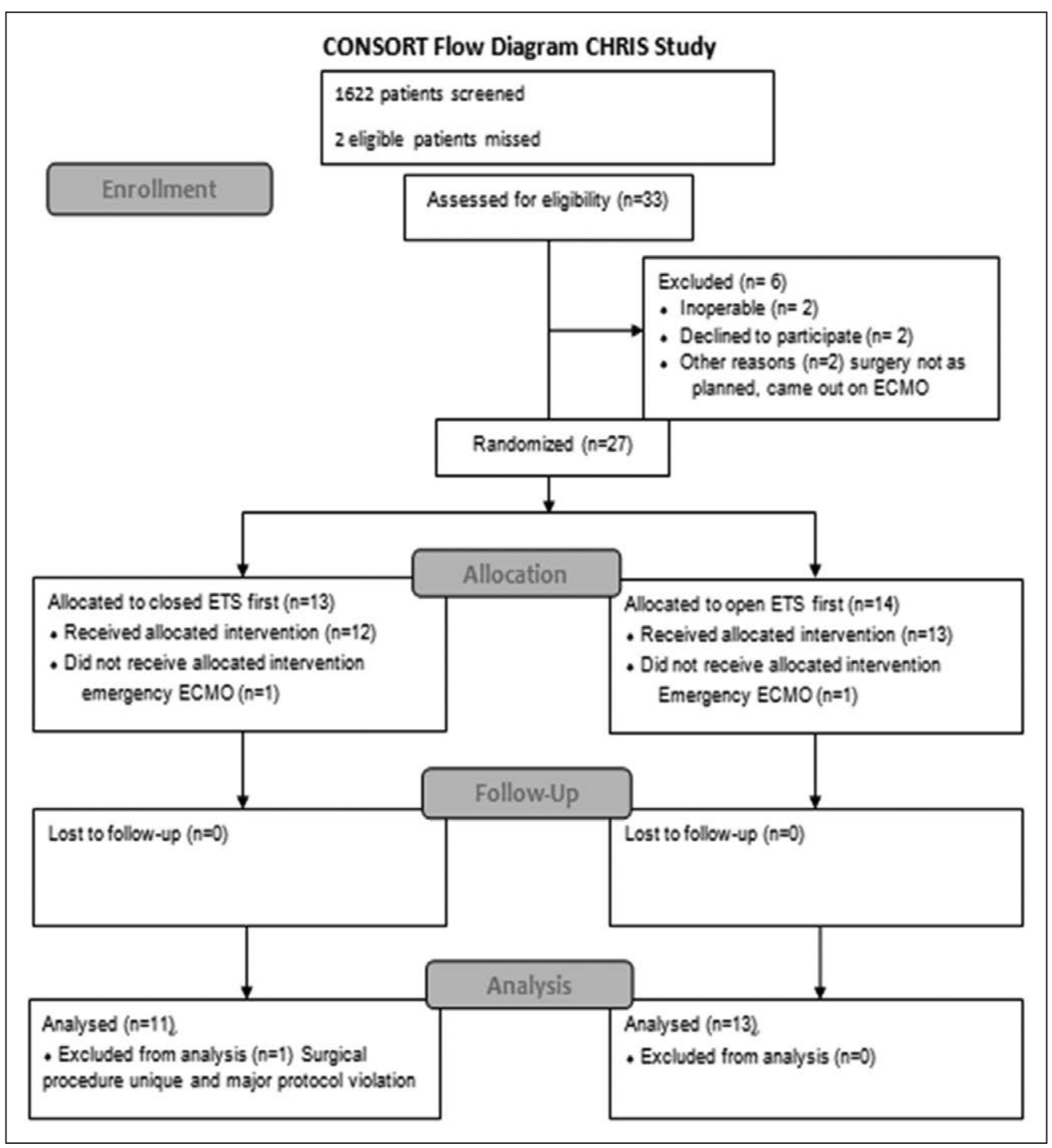

Figure 1. Study Consolidated Standards of Reporting Trials (CONSORT) diagram. CHRIS = Cardiac High Risk Infants Suction Study, ECMO = extracorporeal membrane oxygenation, ETS = tracheal suctioning.

Table 1. Thirteen infants had true single ventricle anatomy, but all had either NS $(n=7)$, MBTS $(n=11)$, or PAB $(n=6)$ surgical procedures. Thirteen patients received the open suction method first followed by the closed suction method second, and 11 patients received the closed suction method first followed by the open suction method second in the first 36 hours after their surgery. The median washout period and time between the two study suctions was 5 hours (IQR, 4-16.2 hr). Tables 2-4 show patient characteristics, drug doses, baseline arterial blood gases, and baseline mechanical ventilation settings prior to the two study measurements. Only one infant crossed over between groups, having been randomized to receive closed suction first, received open suction first, and the second suction was started as closed but crossed over to open due to a large volume of secretions. This patient was analyzed according to the group it was randomized to as per intention to treat.

The mean number of suction passes was 1.5 (total, 1-2 passes), with median saline

the within patient stratum. Once confirmed that the interaction was not significant and that suction order had no impact, the overall means for open and closed methods were computed together with their SEs. Treatment means and SEs at baseline, during, and postprocedure were computed. Two-tailed tests were used, and the level of significance was set at 0.05 . Adjustments to significance levels for multiple testing were not applied, but interpretation of $p$ values was carried out in context as recommended by the American Statistical Association guidance (12). Analysis was undertaken using IBM SPSS software (IBM Analytics, Chicago, IL) and GenStat software (GenStat VSN International, Hemel Hempstead, United Kingdom) (13). Data were analyzed as per intention to treat.

\section{RESULTS}

Twenty-four infants were included in the final analysis (Consolidated Standards of Reporting Trials diagram [Fig. 1]). The median age at surgery was 15 days (interquartile range [IQR], 5-53) with a median weight $3.26 \mathrm{~kg}$ (IQR, 2.8-3.6) volume used $0 \mathrm{~mL}$. Recovery to baseline values was rapid in all infants (median, $0 \mathrm{~min}$ ) with no difference between suction methods. In the pressure-cycled ventilation mode (BIPAP), the preservation of the child's tidal volume at the end of suctioning was not different between the open or closed suction method $(p=0.352)$. No infant suffered any SAE at the time of the ETS measurements undertaken for the study. Table 5 summarizes descriptively the changes in variables between open and closed suctioning.

There was no evidence of any interaction between the order of application and the suction method in the mean change from baseline to peak and baseline to postsuction. The difference between suction methods in mean change from baseline to postsuction was statistically significant for heart rate $(p=0.002)$, SBP $(p=0.022)$, diastolic blood pressure (DBP) $(p=0.009)$, mean blood pressure (MBP) $(p=0.007)$, and arterial saturation ( $p=0.040$ ) with open suctioning demonstrating greater mean changes (Figs. 2 and $\mathbf{3}$ ). There were also statistically significant differences between suction methods in mean 


\section{TABLE 1. Tracheal Suctioning Procedure for High-Risk Cardiac Infants}

Study Variable

1. Administer an additional bolus of IV fentanyl and muscle relaxant (standard doses as prescribed) "2 min prior" to planned procedure.

2. Get equipment ready, wash hands, and have second experienced nurse available to hand bag if required, note baseline patient variables before starting and increase $\mathrm{FIO}_{2}$ on the ventilator by $5 \%$ above the infant's settings at the start of the procedure.

3. Use either open or closed suctioning. If using closed suctioning, the infant is not disconnected from the ventilator, or if using open suction, there is disconnection from the ventilator at this point.

4. Shallow suction (only to the length of the ETT) with a suction catheter size no more than 50\% of the ETT, e.g., for a $3.0 \mathrm{~mm}$ ETT, a size 6 suction catheter, and for a size $3.5 \mathrm{~mm}$ ETT, a size 7 suction catheter, with a suction pressure not exceeding $150 \mathrm{~mm} \mathrm{Hg}$ (but dependent on secretion tenacity).

5. Only apply negative pressure (suction) once the suction catheter at desired length and the whole procedure must take no longer than $10 \mathrm{~s}$.

6. If marked desaturation occurs, increase the $\mathrm{FIO}_{2}$ on the ventilator or get the second experienced nurse to hand ventilate the infant very carefully keeping end-tidal $\mathrm{CO}_{2}$ at a near baseline level while attempting to maintain baseline arterial oxygen saturation levels.

7. "Do not instil normal saline routinely" without prior assessment, only use saline if secretions are thick and it is required.

8. Keep the procedure as quick as possible to as few suction catheter passes as is necessary to clear secretions, and record and document the results of the suction and the infant's response to the procedure.

$\mathrm{ETT}=$ endotracheal tube

\section{TABLE 2. Summary Statistics for Patients' Demographic and Clinical Characteristics}

\begin{tabular}{|c|c|}
\hline Patients Characteristics & Summary Statistics \\
\hline Sex (male), \% & 50 \\
\hline Age at surgery (d), median (IOR) & $15(5-53)$ \\
\hline Weight $(\mathrm{kg})$, median (IOR) & $3.3(2.8-3.6)$ \\
\hline \multicolumn{2}{|l|}{ Surgical procedure, $n$} \\
\hline Norwood Sano & 7 \\
\hline Pulmonary artery banding & 6 \\
\hline Modified Blalock-Taussig shunt & 11 \\
\hline \multicolumn{2}{|l|}{ Endotracheal tube size $(\mathrm{mm}), n(\%)$} \\
\hline 3.0 & $13(54)$ \\
\hline 3.5 & $11(4)$ \\
\hline Cuffed endotracheal tube & $14(59)$ \\
\hline $\begin{array}{l}\text { Day } 1 \text { Pediatric Organ Dysfunction } \\
\text { Score, median (IQR) }\end{array}$ & $40.5(33.2-41.7)$ \\
\hline $\begin{array}{l}\text { Day } 1 \text { Maximal Inotrope Score, } \\
\text { median (IOR) }\end{array}$ & $14.0(9.2-32.5)$ \\
\hline Length of ventilation $(d)$, median (IOR) & $5.0(3.2-8.5)$ \\
\hline PICU length of stay $(d)$, median (IOR) & $8.5(4.0-11.5)$ \\
\hline Mortality at $30 \mathrm{~d}$, alive, $n(\%)$ & $24(100)$ \\
\hline \multicolumn{2}{|l|}{$\begin{array}{l}\text { Serious adverse events in first } 48 \mathrm{hr} \text {, } \\
\quad n(\%)\end{array}$} \\
\hline Cardiopulmonary arrest & $4 / 24(17)$ \\
\hline Emergency chest re-exploration & $2 / 24(8)$ \\
\hline $\begin{array}{l}\text { Acute kidney injury (required peritoneal } \\
\text { dialysis) }\end{array}$ & $5 / 24(21)$ \\
\hline
\end{tabular}

$\mathrm{IQR}=$ interquartile range

\section{TABLE 3. Baseline Drug Data Before Tracheal Suction}

\begin{tabular}{|c|c|c|}
\hline Factors & Drug & $\begin{array}{c}\text { Median } \\
\text { dose (IQR), } \\
\mu \mathrm{g} / \mathrm{kg} / \mathrm{hr}\end{array}$ \\
\hline Opiate infusion & $100 \%$ fentanyl & $1.6(1.5-2)$ \\
\hline Sedative infusion & $100 \%$ midazolam & $40(30-50)$ \\
\hline $\begin{array}{l}\text { Muscle relaxant } \\
\text { infusion }\end{array}$ & $\begin{array}{c}68 \% \text { (32\% IV bolus } \\
\text { relaxants) }\end{array}$ & \\
\hline On inotrope infusion & $77 \%$ & \\
\hline
\end{tabular}

\section{TABLE 4. Baseline Arterial Blood Gases and Mechanical Ventilation Settings}

\section{Baseline Gases and Settings}

Median (IQR)

Arterial blood gases

$\mathrm{pH}$

$7.35(7.28-7.41)$

$\mathrm{PaCO}_{2}(\mathrm{~mm} \mathrm{Hg})$

44.6 (36.3-49.2)

End-tidal $\mathrm{Co}_{2}(\mathrm{~mm} \mathrm{Hg})$

36 (30-42)

Serum lactate ( $\mathrm{mmol} / \mathrm{L})$

$1.7(1.2-2.5)$

Mechanical ventilation settings

$\mathrm{FIO}_{2}(\%)$

$33(21-50)$

Peak inspiratory pressure $(\mathrm{cm})$

$20(18-22)$

Positive end-expiratory pressure $(\mathrm{cm})$

$5(5-5)$

Mean airway pressure $(\mathrm{cm})$

$10(9-10.7)$

Rate (breaths/min)

$30(25-35)$

Inspiratory time (s)

$0.7(0.6-0.75)$ 
TABLE 5.Variable Values Pre- and Postprocedure for Open Suction and Closed Suction

\begin{tabular}{|c|c|c|}
\hline & Closed Suction & Open Suction \\
\hline Study Variable & Median (IQR) & Median (IQR) \\
\hline Recovery time to baseline (min) & $0(0-2)$ & $0(0-2)$ \\
\hline \multicolumn{3}{|c|}{ Echocardiographic flow velocity $(\mathrm{m} / \mathrm{s})$} \\
\hline Baseline & $2.30(1.86-2.62)$ & $2.15(1.93-2.46)$ \\
\hline Postprocedure & $2.23(1.93-2.70)$ & $2.14(1.99-2.51)$ \\
\hline \multicolumn{3}{|l|}{ Heart rate (beats/min) } \\
\hline Baseline & $154.0(132.5-167.3)$ & $151.0(134.5-163.5)$ \\
\hline Postprocedure & $153.5(137.5-168.3)$ & $145.5(139.3-160.5)$ \\
\hline \multicolumn{3}{|l|}{ Systolic BP (mm Hg) } \\
\hline Baseline & $75.0(69.8-81.0)$ & $76.0(65.8-81.0)$ \\
\hline Postprocedure & $75.0(68.0-84.0)$ & $78.5(72.0-84.0)$ \\
\hline \multicolumn{3}{|l|}{ Diastolic BP (mm Hg) } \\
\hline Preprocedure & $40.5(32.0-44.0)$ & $38.0(32.8-40.3)$ \\
\hline Postprocedure & $39.0(31.8-47.0)$ & $41.0(37.0-45.5)$ \\
\hline \multicolumn{3}{|l|}{ Mean BP $(\mathrm{mm} \mathrm{Hg})$} \\
\hline Baseline & $52.5(47.3-58.0)$ & $50.5(46.0-55.0)$ \\
\hline Postprocedure & $52.5(48.5-59.3)$ & $55.0(50.0-58.3)$ \\
\hline \multicolumn{3}{|l|}{ Near infrared spectroscopy (\%) } \\
\hline Baseline & $63.5(58.0-65.0)$ & $60.5(56.5-64.5)$ \\
\hline Postprocedure & $63.5(58.8-67.0)$ & $62.0(56.8-70.0)$ \\
\hline \multicolumn{3}{|l|}{ Arterial oxygen saturation (\%) } \\
\hline Baseline & $77.0(71.5-83.0)$ & $80.5(75.8-83.0)$ \\
\hline Postprocedure & $78.5(70.3-85.3)$ & $80.0(77.5-87.5)$ \\
\hline \multicolumn{3}{|l|}{ Arterial saturation (\%) } \\
\hline Baseline & $81.0(72.5-83.0)$ & $80.5(75.2-83.0)$ \\
\hline Postprocedure & $80.0(73.0-85.7)$ & $81.0(78.0-86.5)$ \\
\hline \multicolumn{3}{|l|}{ End-tidal $\mathrm{PaCO}_{2}(\mathrm{~mm} \mathrm{Hg})$} \\
\hline Baseline & $32.0(28.0-39.5)$ & $31.5(27.2-36.0)$ \\
\hline Postprocedure & $30.0(28.0-41.0)$ & $30.0(27.0-37.0)$ \\
\hline
\end{tabular}

$\mathrm{BP}=$ blood pressure, IQR $=$ interquartile range.

change from baseline to during the suction in $\operatorname{DBP}(p=0.009)$ and MBP $(p=0.029)$ with open suctioning showing greater mean changes. However, clinically none of these changes were significant and did not require any intervention. The largest difference in suction method, measured in terms of mean change from baseline to peak or postsuction, was 4.2 for DBP baseline to post. All other differences had smaller magnitude in the relevant units of measurement for each variable (see Figs. 2 and 3 for the relevant units). Changes in cerebral NIRS were minimal during suction and not different $(p=0.668)$ between suction methods. Fifteen patients (62\%) had echocardiographic measurements done during suctioning. Although in nearly all patients $(13 / 15,86 \%)$ shunt flow velocity or flow velocity across the band reduced, by a mean of $-7.52 \%$ during the suctioning procedure, the recovery to baseline was rapid (all were back to baseline at the postsuction measurement), and there was no difference between suction methods $(p=0.379)$. This varied slightly by surgery type; the NS infants $(n=7)$ showed a mean $-5.9 \%$ reduction from baseline, the MBTS infants $(n=5)$ showed a $-6.5 \%$ reduction from baseline, and the PAB infants $(n=3)$ showed a greater $-12.6 \%$ reduction from baseline flow during ETS. Clinically, there was no significant difference between the two suctioning methods when undertaken as part of this study in controlled conditions 


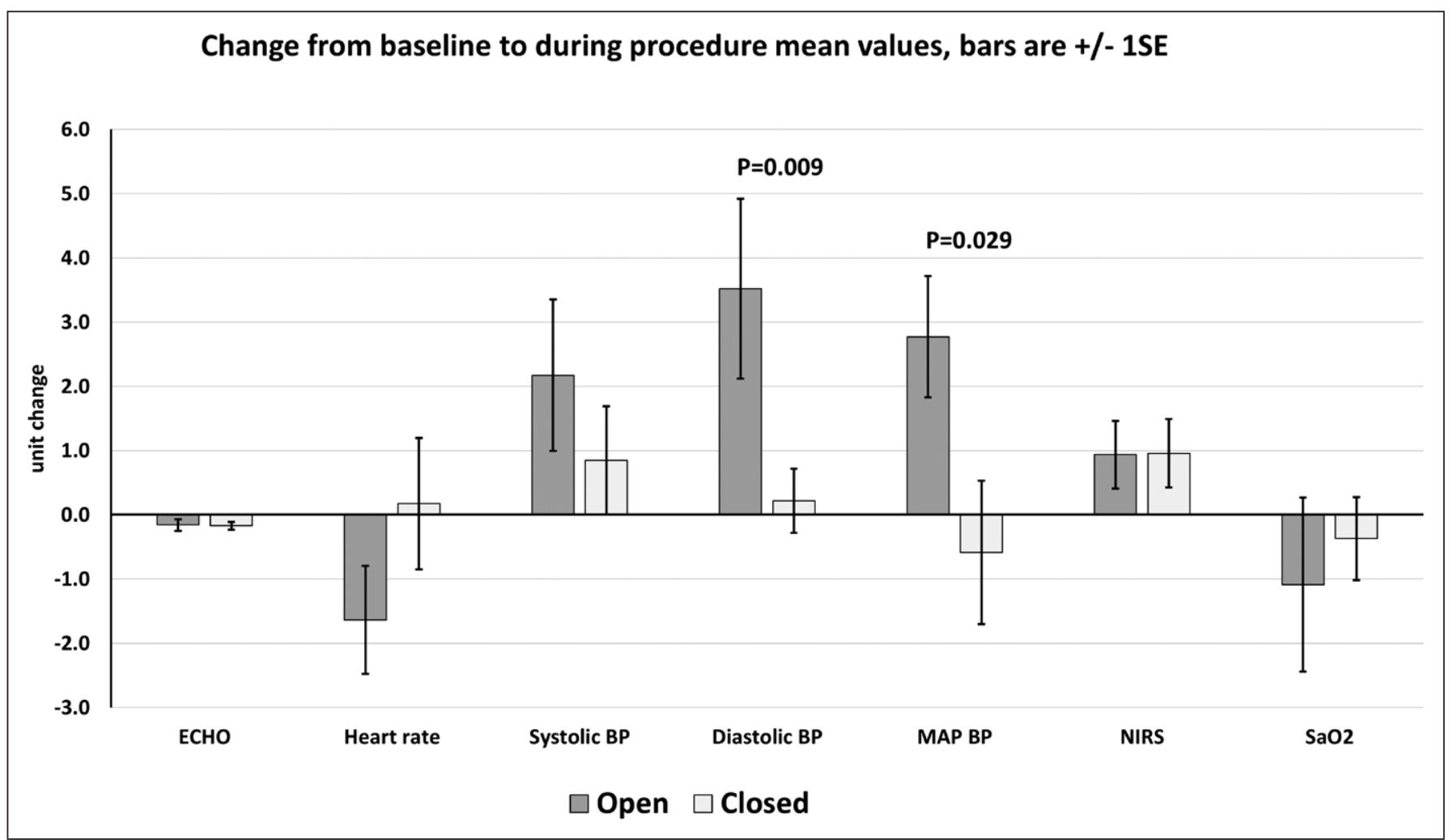

Figure 2. Unit change in physiologic values from baseline to during tracheal suctioning. $\mathrm{BP}=$ blood pressure, ECHO =echocradiographic flow velocity, $\mathrm{MAP}=$ mean arterial pressure, NIRS $=$ near infrared spectroscopy, $\mathrm{SaO}_{2}=$ arterial oxygen saturation.

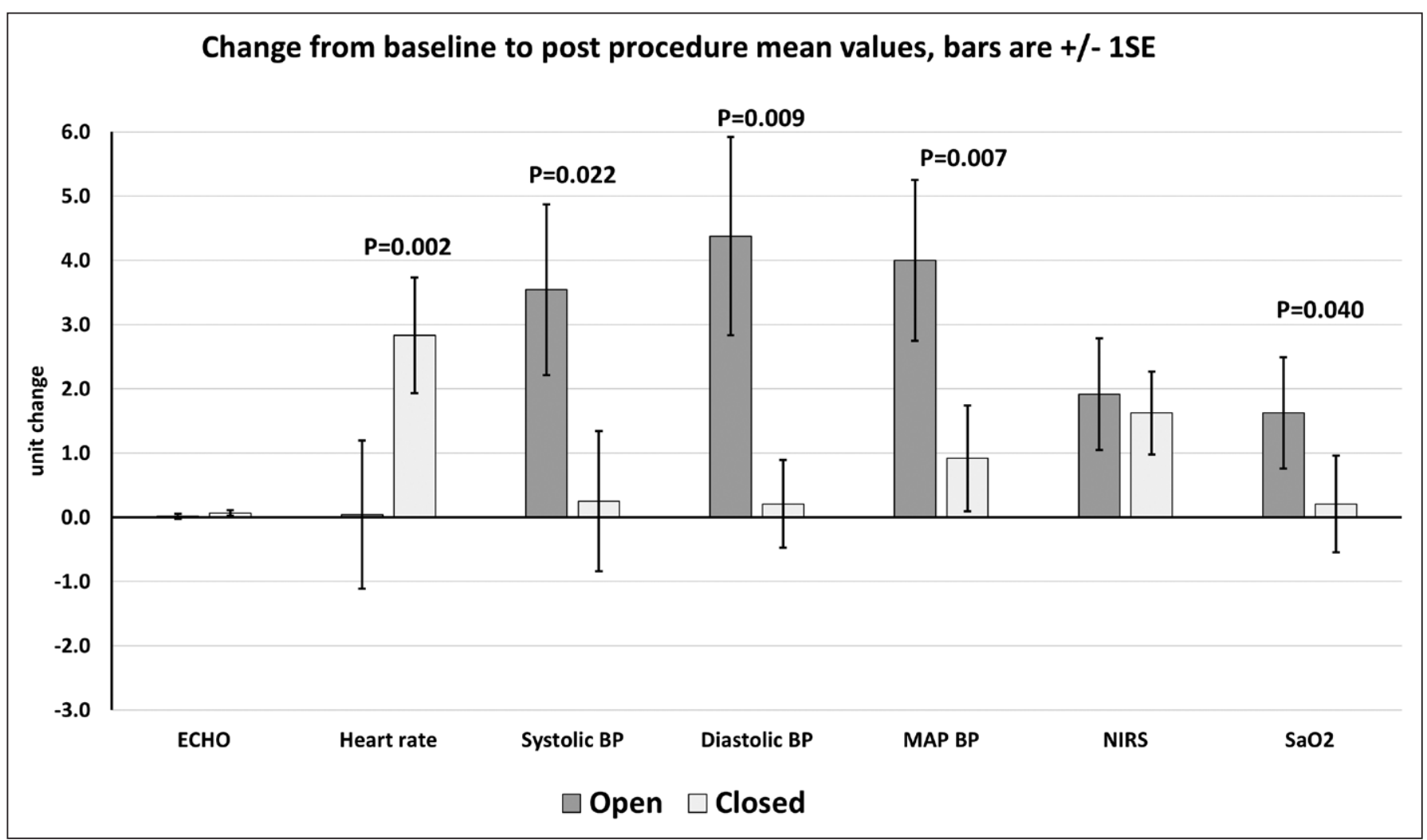

Figure 3. Unit change in physiologic values from baseline to posttracheal suctioning. $\mathrm{BP}=$ blood pressure, MAP $=$ mean arterial pressure, NIRS $=$ near infrared spectroscopy, $\mathrm{SaO}_{2}=$ arterial oxygen saturation. 
(Figs. 2 and 3), although there were greater changes in hemodynamic variables during open suctioning.

\section{DISCUSSION}

This is the first study to specifically examine the impact of ETS method on hemodynamic stability in single ventricle infants after first-stage surgery. There has been other research examining the impact of ETS in other high-risk children: preterm infants (14), children with respiratory disease, (15) and children with traumatic brain injury (16), which have demonstrated the potentially deleterious effects of ETS. The only published work involving tracheal suction in children with congenital heart disease used ETS as a noxious trigger to examine the effect of high-dose fentanyl to blunt the stress response on the pulmonary circulation in infants (17). These infants are at higher risk for hemodynamic instability and CPA.

We found that with experienced study staff, following our PICU tracheal suction guidelines for these patients, there was no clinically significant difference between the two suction methods. There were, however, greater changes in hemodynamic variables with open suctioning, likely to reflect the variability in manual ventilation technique, which is difficult to control. The impact of this is likely to be even more variable in less experienced hands, and in a secondary analysis of routine bedside nurse suctioning in these same infants over the first 48-hour period has demonstrated this (L.N. Tume, et al, unpublished observations, 2017). In this, 211 bedside nurse suctioning episodes (separate to our study measurements) were recorded in these 24 infants. A SAE occurred in 9\% (19/211) of these ETS episodes in 10 infants. PICU unit guidance for suctioning these patients was not always followed, primarily with regard to administration of additional fentanyl and muscle relaxant IV boluses prior to suctioning. Nurses could choose their preferred suction method for their routine suctions, and 74\% (14/19) of these adverse events occurred with open suction, fewer, $26 \%$ $(5 / 19)$ occurred with closed suction. This supports the suggestion that closed suction is likely to be safer in less experienced hands across the whole nursing team.

There are a number of limitations in this study that we acknowledge. This was a pragmatically difficult study in a group of high-risk cardiac patients. We were not able to get echocardiographic data on all 24 infants due to cardiologist availability at the time of suction. One child crossed over between groups, and this child was analyzed according to its randomization group; we did initially analyze the data excluding this child with same results, but as per intention-to-treat principle, we have included them in the analysis. In addition, another child in whom the study guideline for an additional relaxant bolus was not followed was also included, as they exhibited no cough on suction and thus was assumed to be muscle relaxed and included. The time period between study suctions was not controlled and was a pragmatic decision based on the availability of study staff within the designated 36-hour time frame. This may have had an impact on our findings, but this was unrealistic to control for with these highly unstable infants returning from the operating room at different times of the day or night. Despite these limitations, this is the first study to examine the specific impact of ETS type in high-risk cardiac infants with unstable pulmonary to systematic blood flow, and we believe the findings to be important.

Further research should be directed at exploring how experienced "expert" pediatric cardiac intensive care nurses plan for and mitigate risk during ETS in these high-risk children. If we could articulate clearly the expert nurses' thinking and actions, this may be able to be developed in "teachable" clinical competencies so that less experienced nurses could be instructed, rather than having to solely "learn on the job" by trial and error.

\section{CONCLUSIONS}

ETS cannot be avoided in intubated children and in children at greater risk of hemodynamic instability such as these infants. It is important to generate evidence to determine the safest ETS method and to quantify the hemodynamic impact of this essential procedure. Our study showed no clinically significant differences between closed or open ETS in experienced hands; however, the greater changes seen in physiologic variables with open suctioning, suggests that if guidelines are to be developed for use across a whole ICU, it would be safer to recommend closed suction. Much of pediatric cardiac intensive care nursing practice is based on experience and intuition, rather than evidence, and this can only be addressed through further research.

\section{ACKNOWLEDGMENT}

We thank the Children's Heart Federation parent group (based at Alder Hey Hospital) for reviewing the study and providing feedback on the parent information sheets and all the clinical staff at Alder Hey Children's Hospital who participated in this study.

\section{REFERENCES}

1. Congenital Heart Disease Website With Data Collected by the Central Cardiac Audit Database (CCAD) Congenital Heart Disease Registry in UK. Available at: http://nicor4.nicor.org.uk/chd/an_paeds. nsf/wcontent/home. Accessed November 24, 2016

2. Morrow BM, Argent AC: A comprehensive review of pediatric endotracheal suctioning: Effects, indications, and clinical practice. Pediatr Crit Care Med 2008; 9:465-477

3. Gardner DL, Shirland L: Evidence-based guideline for suctioning the intubated neonate and infant. Neonatal Netw 2009; 28:281-302

4. American Association for Respiratory Care: AARC Clinical Practice Guidelines. Endotracheal suctioning of mechanically ventilated patients with artificial airways 2010. Respir Care 2010; 55:758-764

5. Tume LN, Copnell B: Endotracheal suctioning of the critically ill child. J Pediatr Intensive Care 2015; 4:56-63

6. Hoellering AB, Copnell B, Dargaville PA, et al: Lung volume and cardiorespiratory changes during open and closed endotracheal suction in ventilated newborn infants. Arch Dis Child Fetal Neonatal Ed 2008; 93:F436-F441

7. Taylor JE, Hawley G, Flenady V, et al: Tracheal suctioning without disconnection in intubated ventilated neonates. Cochrane Database Syst Rev 2011; 12:CD003065

8. Tingay DG, Copnell B, Mills JF, et al: Effects of open endotracheal suction on lung volume in infants receiving HFOV. Intensive Care Med 2007; 33:689-693 
9. Copnell B, Tingay DG, Kiraly NJ, et al: A comparison of the effectiveness of open and closed endotracheal suction. Intensive Care Med 2007; 33:1655-1662

10. Kiraly NJ, Tingay DG, Mills JF, et al: Volume not guaranteed: Closed endotracheal suction compromises ventilation in volume-targeted mode. Neonatology 2011; 99:78-82

11. Sealed Envelope Software. Available at: https://www.sealedenvelope. com/. Accessed August 1, 2014

12. Wasserstein RL, Lazar NA: The ASA's statement on p values: Context, process and purpose. Am Stat 2016; 70:129-133

13. GenStat Statistical Analysis Software. Available at: https://www.vsni. co.uk/software/genstat/. Accessed June 1, 2016
14. Durand M, Sangha B, Cabal LA, et al: Cardiopulmonary and intracranial pressure changes related to endotracheal suctioning in preterm infants. Crit Care Med 1989; 17:506-510

15. Morrow B, Futter M, Argent A: Effect of endotracheal suction on lung dynamics in mechanically-ventilated paediatric patients. Aust $J$ Physiother 2006; 52:121-126

16. Tume LN, Baines PB, Lisboa PJ: The effect of nursing interventions on the intracranial pressure in paediatric traumatic brain injury. Nurs Crit Care 2011; 16:77-84

17. Hickey PR, Hansen DD, Wessel DL, et al: Blunting of stress responses in the pulmonary circulation of infants by fentanyl. Anesth Analg 1985; 64:1137-1142 